\title{
A Class of Iterative Nonlinear Difference Inequality with Weakly Singularity
}

\author{
Chunmiao Huang, ${ }^{1}$ Wu-Sheng Wang, ${ }^{1}$ and Xiaoliang Zhou ${ }^{2}$ \\ ${ }^{1}$ School of Mathematics and Statistics, Hechi University, Yizhou, Guangxi 546300, China \\ ${ }^{2}$ Department of Mathematics, Zhanjiang Normal University, Zhanjiang, Guangdong 524088, China
}

Correspondence should be addressed to Wu-Sheng Wang; wang4896@126.com

Received 27 March 2014; Accepted 28 April 2014; Published 8 May 2014

Academic Editor: Junjie Wei

Copyright (c) 2014 Chunmiao Huang et al. This is an open access article distributed under the Creative Commons Attribution License, which permits unrestricted use, distribution, and reproduction in any medium, provided the original work is properly cited.

We discuss a class of new nonlinear weakly singular difference inequality, which is solved by change of variable, discrete Hölder inequality, discrete Jensen inequality, the mean-value theorem for integrals and amplification method, and Gamma function. Explicit bound for the unknown function is given clearly. Moreover, an example is presented to show the usefulness of our results.

\section{Introduction}

Being an important tool in the study of qualitative properties of solutions of differential equations and integral equations, various generalizations of Gronwall inequalities and their applications have attracted great interests of many mathematicians (such as [1-21]). Gronwall-Bellman inequality [22, 23 ] can be stated as follows: if $u$ and $f$ are nonnegative and continuous functions on an interval $[a, b]$ satisfying

$$
u(t) \leq c+\int_{a}^{t} f(s) u(s) d s, \quad t \in[a, b],
$$

for some constant $c \geq 0$, then

$$
u(t) \leq c \exp \left(\int_{a}^{t} f(s) d s\right), \quad t \in[a, b] .
$$

In 1981, Henry [2] discussed the following linear singular integral inequality:

$$
u(t) \leq a+b \int_{0}^{t}(t-s)^{\beta-1} u(s) d s .
$$

In 2007, Ye et al. [20] discussed linear singular integral inequality:

$$
u(t) \leq a(t)+b(t) \int_{0}^{t}(t-s)^{\beta-1} u(s) d s .
$$

In 2011, Abdeldaim and Yakout [21] studied a new integral inequality of Gronwall-Bellman-Pachpatte type

$$
\begin{aligned}
& u(t) \leq u_{0}+\int_{t_{0}}^{t} f(s) u(s) \\
& \times\left[u(s)+\int_{t_{0}}^{s} h(\tau)\right. \\
&\left.\quad \times\left[u(\tau)+\int_{t_{0}}^{\tau} g(\xi) u(\xi) d \xi\right] d \tau\right] d s .
\end{aligned}
$$

On the other hand, many physical problems arising in a wide variety of applications are governed by finite difference equations. The theory of difference equations has been developed as a natural discrete analogue of corresponding theory of differential equations. Difference inequalities which give explicit bounds on unknown functions provide a very useful and important tool in the study of many qualitative as well as quantitative properties of solutions of nonlinear difference equations (such as [24-32]). Sugiyama [26] established the most precise and complete discrete analogue of the Gronwall inequality in the following form:

$$
u(n) \leq u_{0}+\sum_{s=n_{0}}^{n-1} f(s) u(s) .
$$


For instance, Pachpatte [27] considered the following discrete inequality:

$$
\begin{aligned}
u(n) \leq & u_{0}+\sum_{s=n_{0}}^{n-1} f(s)[u(s)+h(s)] \\
& +\sum_{s=n_{0}}^{n-1} f(s)\left(\sum_{\tau=n_{0}}^{s-1} g(\tau) u(\tau)\right), \quad \forall n \in N_{0} .
\end{aligned}
$$

In 2006, Cheung and Ren [29] studied

$$
\begin{aligned}
u^{p}(m, n) \leq & c+\sum_{s=m_{0}}^{m-1} \sum_{t=n_{0}}^{n-1} a(s, t) u^{q}(s, t) \\
& +\sum_{s=m_{0}}^{m-1} \sum_{t=n_{0}}^{n-1} b(s, t) u^{q}(s, t) w(u(s, t)) .
\end{aligned}
$$

Later, Zheng et al. [31] discussed the following discrete inequality:

$$
u(n) \leq a(n)+\sum_{i=1}^{k} \sum_{s=0}^{n-1} f_{i}(n, s) w_{i}(u(s)) .
$$

Motivated by the results given in $[2,20,21,32]$, in this paper, we discuss a new linear singular integral inequality

$$
\begin{aligned}
u(n) \leq & a(n)+b(n) \\
& \times \sum_{s=0}^{n-1}\left(t_{n}-t_{s}\right)^{\beta-1} \tau_{s} w_{1}(u(s)) \\
& \times\left[u(s)+h(s)+\sum_{\tau=0}^{s-1}\left(t_{s}-t_{\tau}\right)^{\beta-1} \tau_{\tau} w_{2}(u(\tau))\right],
\end{aligned}
$$

where $t_{0}=0, \tau_{s}=t_{s+1}-t_{s}>0, \sup _{s \in \mathbf{N}, 0 \leq s \leq n-1}\left\{\tau_{s}, s \in \mathbf{N}\right\}=\tau$, and $\lim _{n \rightarrow \infty} t_{n}=\infty$.

For the reader's convenience, we present some necessary lemmas.

Lemma 1 (discrete Jensen inequality [28]). Let $A_{1}, A_{2}, \ldots, A_{n}$ be nonnegative real numbers, $r>1$ is a real number, and $n$ is a natural number. Then

$$
\left(A_{1}+A_{2}+\cdots+A_{n}\right)^{r} \leq n^{r-1}\left(A_{1}^{r}+A_{2}^{r}+\cdots+A_{n}^{r}\right) .
$$

Lemma 2 (discrete Hölder inequality [30]). Let $a_{i}, b_{i}(i=$ $1,2, \ldots, n)$ be nonnegative real numbers, and let $p, q$ be positive numbers such that $(1 / q)+(1 / p)=1$; then

$$
\sum_{s=0}^{n-1} a_{i} b_{i} \leq\left(\sum_{s=0}^{n-1} a_{i}^{p}\right)^{1 / p}\left(\sum_{s=0}^{n-1} b_{i}^{q}\right)^{1 / q}
$$

Lemma 3. Let $t_{0}=0, \tau_{s}=t_{s+1}-t_{s}>0, \sup _{s \in \mathrm{N}, 0 \leq s \leq n-1}\left\{\tau_{s}, s \in\right.$ $\mathbf{N}\}=\tau$, and $\lim _{n \rightarrow \infty} t_{n}=\infty$. If $\beta \in[0,1 / 2], 0<p<1 /(1-\beta)$, then

$$
\sum_{s=0}^{n-1}\left(t_{n}-t_{s}\right)^{p(\beta-1)} e^{p t_{s}} \tau_{s} \leq \frac{e^{p t_{n}}}{p^{1+p(\beta-1)}} \Gamma(1+p(\beta-1)),
$$

where $1+p(\beta-1)>0, \Gamma(\beta):=\int_{0}^{\infty} \tau^{\beta-1} e^{-\tau} d \tau$ is the well-known $\Gamma$-function.

Proof. By the definition of integration and the conditions in Lemma 3, we have

$$
\sum_{s=0}^{n-1}\left(t_{n}-t_{s}\right)^{p \beta-p} e^{p t_{s}} \tau_{s} \leq \int_{0}^{t_{n}}\left(t_{n}-s\right)^{p \beta-p} e^{p s} d s
$$

Using a change of variables $\tau=t_{n}-s$ and $\xi=p \tau$, we have the estimation

$$
\begin{aligned}
\int_{0}^{t_{n}}\left(t_{n}-s\right)^{p(\beta-1)} e^{p s} d s & =-\int_{t_{n}}^{0} \tau^{p(\beta-1)} e^{p t_{n}-p \tau} d \tau \\
& =e^{p t_{n}} \int_{0}^{t_{n}} \tau^{p(\beta-1)} e^{-p \tau} d \tau \\
& =\frac{e^{p t_{n}}}{p^{1+p(\beta-1)}} \int_{0}^{p t_{n}} \xi^{p(\beta-1)} e^{-\xi} d \xi \\
& \leq \frac{e^{p t_{n}}}{p^{1+p(\beta-1)}} \Gamma(1+p(\beta-1)) .
\end{aligned}
$$

Since $0<\beta \leq 1 / 2, p<1 /(1-\beta), 1+p(\beta-1)>0$, and $\Gamma(1+p(\beta-1)) \in R^{+}$, from (14) and (15), we have the relation (13).

\section{Main Result}

In this section, we give the estimation of unknown function in (10). Let $\mathbf{N}:=\{0,1,2, \ldots\}$. For function $z(n)$, its difference is defined by $\Delta z=z(n+1)-z(n)$. Obviously, the linear difference equation $\triangle z(n)=b(n)$ with the initial condition $z\left(n_{0}\right)=0$ has the solution $z(n)=\sum_{s=0}^{n-1} b(s)$. For convenience, in the sequel we complementarily define that $\sum_{s=0}^{-1} b(s)=0$.

Theorem 4. Suppose that $0<\beta \leq 1 / 2$ is a constant, $a(n), b(n)$ are nonnegative and nondecreasing functions defined on $\mathbf{N}, w_{1}(u), w_{2}(u) / w_{1}(u), w_{2}^{q}\left(u^{1 / q}\right) /\left(u w_{1}^{q}\left(u^{1 / q}\right)\right)$ are nonnegative, nondecreasing, and continuous functions defined on $\mathbf{R}_{+}$, $t_{0}=0, \tau_{s}=t_{s+1}-t_{s}>0, \sup _{s \in \mathbf{N}, 0 \leq s \leq n-1}\left\{\tau_{s}, s \in \mathbf{N}\right\}=\tau$, and $\lim _{n \rightarrow \infty} t_{n}=\infty$. If $u(t)$ satisfies $(10)$, then

$$
u(n) \leq\left\{H_{1}^{-1}\left\{H_{2}^{-1}\left[H_{3}^{-1}(U(n))\right]\right\}\right\}^{1 / q}, \quad \forall n \in \mathbf{N}_{1}
$$


where

$$
\begin{aligned}
& U(n):=H_{3}\left(H_{2}\left(H_{1}\left(2^{q-1} a(n)\right)+f(n) \sum_{s=0}^{n-1} h^{q}(s) e^{-q t_{s}}\right)\right. \\
& \left.+f(n) \sum_{s=0}^{n-1} e^{-q t_{s}}\right)+g(n) \sum_{s=0}^{n-1} e^{-q t_{s}}, \\
& H_{1}(u):=\int_{c_{1}}^{u} \frac{d s}{w_{1}^{q}\left(s^{1 / q}\right)}, \quad u>0, c_{1}>0, \\
& H_{2}(u):=\int_{\mathcal{C}_{2}}^{u} \frac{d s}{H_{1}^{-1}(s)}, \quad u>0, c_{2}>0, \\
& H_{3}(u):=\int_{\mathcal{C}_{3}}^{u} \frac{H_{2}^{-1}\left(H_{1}^{-1}(s)\right) w_{1}^{q}\left(\left(H_{2}^{-1}\left(H_{1}^{-1}(s)\right)\right)^{1 / q}\right)}{w_{2}^{q}\left(\left(H_{2}^{-1}\left(H_{1}^{-1}(s)\right)\right)^{1 / q}\right)} \text {, }
\end{aligned}
$$$$
u>0, c_{3}>0 \text {, }
$$

$$
\begin{gathered}
f(n):=6^{q-1}(b(n))^{q} \tau^{q(p-1) / p} \\
\times\left[\frac{e^{p t_{n}}}{p^{1+p(\beta-1)}} \Gamma(1+p(\beta-1))\right]^{q / p}, \\
g(n):=\tau^{q(p-1) / p}\left(\frac{e^{p t_{n}}}{p^{1+p(\beta-1)}} \Gamma(1+p(\beta-1))\right)^{q / p},
\end{gathered}
$$

and $p=1+\beta, q=1+1 / \beta, \mathbf{N}_{1}:=\left\{0,1,2, \ldots, K_{1}\right\}, K_{1}$ is the largest integer number such that

$$
\begin{gathered}
U\left(K_{1}\right) \in \operatorname{Dom}\left(H_{3}^{-1}\right), \quad H_{3}^{-1}\left(U\left(K_{1}\right)\right) \in \operatorname{Dom}\left(H_{2}^{-1}\right), \\
H_{2}^{-1}\left(H_{3}^{-1}\left(U\left(K_{1}\right)\right)\right) \in \operatorname{Dom}\left(H_{1}^{-1}\right) .
\end{gathered}
$$

Proof. From (10), we have

$$
\begin{aligned}
& u(n) \leq a(n)+b(n) \\
& \times \sum_{s=0}^{n-1}\left(t_{n}-t_{s}\right)^{\beta-1} e^{t_{s}} e^{-t_{s}} \tau_{s} w_{1}(u(s)) \\
& \times\left[u(s)+h(s)+\sum_{\sigma=0}^{s-1}\left(t_{s}-t_{\sigma}\right)^{\beta-1} \tau_{\sigma} w_{2}(u(\sigma))\right], \\
& \forall n \in \mathbf{N} .
\end{aligned}
$$

Applying Lemma 2 with $p=1+\beta, q=1+1 / \beta$ to (24), we obtain that

$$
\begin{aligned}
u(n) \leq & a(n)+b(n) \tau^{(p-1) / p}\left[\sum_{s=0}^{n-1}\left(t_{n}-t_{s}\right)^{p \beta-p} e^{p t_{s}} \tau_{s}\right]^{1 / p} \\
& \times\left[\sum_{s=0}^{n-1} e^{-q t_{s}} w_{1}^{q}(u(s))\right. \\
& \left.\times\left[u(s)+h(s)+\sum_{\sigma=0}^{s-1}\left(t_{s}-t_{\sigma}\right)^{\beta-1} \tau_{\sigma} w_{2}(u(\sigma))\right]^{q}\right]^{1 / q},
\end{aligned}
$$

where $\tau_{s}<\tau$ is used. Applying Lemma 3, we have

$$
\begin{aligned}
u(n) \leq & a(n)+b(n) \tau^{(p-1) / p}\left[\frac{e^{p t_{n}}}{p^{1+p(\beta-1)}} \Gamma(1+p(\beta-1))\right]^{1 / p} \\
\times & {\left[\sum_{s=0}^{n-1} e^{-q t_{s}} w_{1}^{q}(u(s))\right.} \\
& \left.\times\left[u(s)+h(s)+\sum_{\sigma=0}^{s-1}\left(t_{s}-t_{\sigma}\right)^{\beta-1} \tau_{\sigma} w_{2}(u(\sigma))\right]^{q}\right]^{1 / q} .
\end{aligned}
$$

By discrete Jensen inequality (11) with $n=2, r=q$, from (26) we obtain that

$$
\begin{aligned}
u^{q}(n) \leq & 2^{q-1}(a(n))^{q}+2^{q-1}(b(n))^{q} \tau^{q(p-1) / p} \\
& \times\left[\frac{e^{p t_{n}}}{p^{1+p(\beta-1)}} \Gamma(1+p(\beta-1))\right]^{q / p} \\
& \times \sum_{s=0}^{n-1} e^{-q t_{s}} w_{1}^{q}(u(s)) \\
& \times\left[u(s)+h(s)+\sum_{\sigma=0}^{s-1}\left(t_{s}-t_{\sigma}\right)^{\beta-1} \tau_{\sigma} w_{2}(u(\sigma))\right]^{q} .
\end{aligned}
$$


Again using discrete Jensen inequality (11) with $n=3, r=q$, from (27) we obtain that

$$
\begin{aligned}
u^{q}(n) \leq & 2^{q-1}(a(n))^{q}+2^{q-1}(b(n))^{q} \tau^{q(p-1) / p} \\
& \times\left[\frac{e^{p t_{n}}}{p^{1+p(\beta-1)}} \Gamma(1+p(\beta-1))\right]^{q / p} \\
& \times \sum_{s=0}^{n-1} e^{-q t_{s}} w_{1}^{q}(u(s)) \\
& \times\left[3^{q-1} u^{q}(s)+3^{q-1} h^{q}(s)+3^{q-1}\right. \\
& \left.\quad \times\left[\sum_{\sigma=0}^{s-1}\left(t_{s}-t_{\sigma}\right)^{\beta-1} \tau_{\sigma} w_{2}(u(\sigma))\right]^{q}\right],
\end{aligned}
$$

For $\left[\sum_{\sigma=0}^{s-1}\left(t_{s}-t_{\sigma}\right)^{\beta-1} \tau_{\sigma} w_{2}(u(\sigma))\right]^{q}$ in (28), applying Lemma 2 with $p=1+\beta, q=1+1 / \beta$, we obtain that

$$
\begin{aligned}
& {\left[\sum_{\sigma=0}^{s-1}\left(t_{s}-t_{\sigma}\right)^{\beta-1} \tau_{\sigma} w_{2}(u(\sigma))\right]^{q}} \\
& \leq \tau^{q(p-1) / p}\left(\sum_{\sigma=0}^{s-1}\left(t_{s}-t_{\sigma}\right)^{p \beta-p} e^{p t_{\sigma}} \tau_{\sigma}\right)^{q / p} \\
& \quad \times \sum_{\sigma=0}^{s-1} e^{-q t_{\sigma}} w_{2}^{q}(u(\sigma)) \\
& \leq \tau^{q(p-1) / p}\left(\frac{e^{p t_{s}}}{\left.p^{1+p(\beta-1)} \Gamma(1+p(\beta-1))\right)^{q / p}}\right. \\
& \quad \times \sum_{\sigma=0}^{s-1} e^{-q t_{\sigma}} w_{2}^{q}(u(\sigma))
\end{aligned}
$$

here Lemma 3 is used. Substituting (29) into (28), we have

$$
\begin{aligned}
u^{q}(n) \leq & 2^{q-1}(a(n))^{q}+2^{q-1}(b(n))^{q} \tau^{q(p-1) / p} \\
& \times\left[\frac{e^{p t_{n}}}{p^{1+p(\beta-1)}} \Gamma(1+p(\beta-1))\right]^{q / p} \sum_{s=0}^{n-1} e^{-q t_{s}} w_{1}^{q}(u(s)) \\
& \times\left[3^{q-1} u^{q}(s)+3^{q-1} h^{q}(s)+3^{q-1} \tau^{q(p-1) / p}\right. \\
& \times\left(\frac{e^{p t_{s}}}{p^{1+p(\beta-1)}} \Gamma(1+p(\beta-1))\right)^{q / p} \\
& \left.\times \sum_{\sigma=0}^{s-1} e^{-q t_{\sigma}} w_{2}^{q}(u(\sigma))\right]
\end{aligned}
$$

$$
\begin{aligned}
= & 2^{q-1}(a(n))^{q}+f(n) \sum_{s=0}^{n-1} e^{-q t_{s}} w_{1}^{q}(u(s)) \\
& \times\left[u^{q}(s)+h^{q}(s)+g(s) \sum_{\sigma=0}^{s-1} e^{-q t_{\sigma}} w_{2}^{q}(u(\sigma))\right] \\
= & 2^{q-1}(a(n))^{q}+f(n) \sum_{s=0}^{n-1} h^{q}(s) e^{-q t_{s}} w_{1}^{q}(u(s)) \\
& +f(n) \sum_{s=0}^{n-1} e^{-q t_{s}} w_{1}^{q}(u(s)) \\
& \times\left[u^{q}(s)+g(s) \sum_{\sigma=0}^{s-1} e^{-q t_{\sigma}} w_{2}^{q}(u(\sigma))\right], \\
& \quad \forall n \in \mathbf{N},
\end{aligned}
$$

where $f(n)$ and $g(n)$ are defined by (21) and (22), respectively. Let $v(n):=u^{q}(n)$; from (30) we have

$$
\begin{aligned}
& v(n) \leq 2^{q-1}(a(n))^{q}+f(n) \sum_{s=0}^{n-1} h^{q}(s) e^{-q t_{s}} w_{1}^{q}\left(v^{1 / q}(s)\right) \\
&+f(n) \sum_{s=0}^{n-1} e^{-q t_{s}} w_{1}^{q}\left(v^{1 / q}(s)\right) \\
& \times\left[v(s)+g(s) \sum_{\sigma=0}^{s-1} e^{-q t_{\sigma}} w_{2}^{q}\left(v^{1 / q}(\sigma)\right)\right], \\
& \forall n \in \mathbf{N} .
\end{aligned}
$$

Since $f(n), g(n)$ are nondecreasing functions, from (31) we have

$$
\begin{aligned}
& v(n) \leq 2^{q-1}(a(K))^{q}+f(K) \sum_{s=0}^{n-1} h^{q}(s) e^{-q t_{s}} w_{1}^{q}\left(v^{1 / q}(s)\right) \\
&+f(K) \sum_{s=0}^{n-1} e^{-q t_{s}} w_{1}^{q}\left(v^{1 / q}(s)\right) \\
& \times\left[v(s)+g(K) \sum_{\sigma=0}^{s-1} e^{-q t_{\sigma}} w_{2}^{q}\left(v^{1 / q}(\sigma)\right)\right], \\
& \forall n \in[0, K] \cap \mathbf{N},
\end{aligned}
$$

where $K \in \mathbf{N}, K \leq K_{1}$ is chosen arbitrarily.

Let $z_{1}(t)$ denote the function on the right-hand side of (32), which is a positive and nondecreasing function on $[0, K] \cap \mathbf{N}$. From (32), we have

$z_{1}(0)=2^{q-1}(a(K))^{q}, \quad v(n) \leq z_{1}(n), \quad \forall n \in[0, K] \cap \mathbf{N}$. 
Using $\Delta z_{1}(n)=z_{1}(n+1)-z_{1}(n)$ and (33), we obtain

$$
\begin{aligned}
\Delta z_{1}(n)= & f(K) h^{q}(n) e^{-q t_{n}} w_{1}^{q}\left(v^{1 / q}(n)\right) \\
& +f(K) e^{-q t_{n}} w_{1}^{q}\left(v^{1 / q}(n)\right) \\
& \times\left[v(n)+g(K) \sum_{\sigma=0}^{n-1} e^{-q t_{\sigma}} w_{2}^{q}\left(v^{1 / q}(\sigma)\right)\right] \\
\leq & f(K) h^{q}(n) e^{-q t_{n}} w_{1}^{q}\left(z_{1}^{1 / q}(n)\right) \\
& +f(K) e^{-q t_{n}} w_{1}^{q}\left(z_{1}^{1 / q}(n)\right) \\
& \times\left[z_{1}(n)+g(K) \sum_{\sigma=0}^{n-1} e^{-q t_{\sigma}} w_{2}^{q}\left(z_{1}^{1 / q}(\sigma)\right)\right],
\end{aligned}
$$

for all $n \in[0, K] \cap \mathbf{N}$.

Let

$$
y(n)=z_{1}(n)+g(K) \sum_{\sigma=0}^{n-1} e^{-q t_{\sigma}} w_{2}^{q}\left(z_{1}^{1 / q}(\sigma)\right),
$$

Then

$$
y(0)=z_{1}(0), \quad z_{1}(n) \leq y(n), \quad \forall n \in[0, K] \cap \mathbf{N} .
$$

From (35), we have

$$
\begin{aligned}
\Delta y(n)= & \Delta z_{1}(n)+g(K) e^{-q t_{n}} w_{2}^{q}\left(z_{1}^{1 / q}(n)\right) \\
\leq & f(K) h^{q}(n) e^{-q t_{n}} w_{1}^{q}\left(z_{1}^{1 / q}(n)\right) \\
& +f(K) e^{-q t_{n}} w_{1}^{q}\left(z_{1}^{1 / q}(n)\right) y(n) \\
& +g(K) e^{-q t_{n}} w_{2}^{q}\left(z_{1}^{1 / q}(n)\right) \\
\leq & f(K) h^{q}(n) e^{-q t_{n}} w_{1}^{q}\left(y^{1 / q}(n)\right) \\
& +f(K) e^{-q t_{n}} w_{1}^{q}\left(y^{1 / q}(n)\right) y(n) \\
& +g(K) e^{-q t_{n}} w_{2}^{q}\left(y^{1 / q}(n)\right) .
\end{aligned}
$$

It implies that, for all $n \in[0, K] \cap \mathbf{N}$,

$$
\begin{aligned}
\frac{\Delta y(n)}{w_{1}^{q}\left(y^{1 / q}(n)\right)} \leq & f(K) h^{q}(n) e^{-q t_{n}}+f(K) e^{-q t_{n}} y(n) \\
& +g(K) e^{-q t_{n}} \frac{w_{2}^{q}\left(y^{1 / q}(n)\right)}{w_{1}^{q}\left(y^{1 / q}(n)\right)} .
\end{aligned}
$$

On the other hand, by the mean-value theorem for integrals, for arbitrarily given integers $n, n+1 \in[0, K] \cap \mathbf{N}$, there exists $\xi$ in the open interval $(y(n), y(n+1))$ such that

$$
\begin{aligned}
H_{1}(y(n+1))-H_{1}(y(n)) & =\int_{y(n)}^{y(n+1)} \frac{d s}{w_{1}^{q}\left(s^{1 / q}\right)} \\
& =\frac{\Delta y(n)}{w_{1}^{q}\left(\xi^{1 / q}\right)} \leq \frac{\Delta y(n)}{w_{1}^{q}\left(y^{1 / q}(n)\right)},
\end{aligned}
$$

for all $n \in[0, K] \cap \mathbf{N}$, where $H_{1}$ is defined by (18). From (38) and (39), we have

$$
\begin{aligned}
H_{1}(y(n+1))-H_{1}(y(n)) \leq & f(K) h^{q}(n) e^{-q t_{n}} \\
& +f(K) e^{-q t_{n}} y(n) \\
& +g(K) e^{-q t_{n}} \frac{w_{2}^{q}\left(y^{1 / q}(n)\right)}{w_{1}^{q}\left(y^{1 / q}(n)\right)} .
\end{aligned}
$$

Taking $n=s$ in (40) and summing up over $s$ from 0 to $n-1$, from (40) we obtain

$$
\begin{aligned}
& H_{1}(y(n)) \leq H_{1}(y(0))+\sum_{s=0}^{n-1} f(K) h^{q}(s) e^{-q t_{s}} \\
&+\sum_{s=0}^{n-1} f(K) e^{-q t_{s}} y(s) \\
&+\sum_{s=0}^{n-1} g(K) e^{-q t_{s}} \frac{w_{2}^{q}\left(y^{1 / q}(s)\right)}{w_{1}^{q}\left(y^{1 / q}(s)\right)} \\
& \leq H_{1}(y(0))+\sum_{s=0}^{K-1} f(K) h^{q}(s) e^{-q t_{s}} \\
&+\sum_{s=0}^{n-1} f(K) e^{-q t_{s}} y(s) \\
&+\sum_{s=0}^{n-1} g(K) e^{-q t_{s}} \frac{w_{2}^{q}\left(y^{1 / q}(s)\right)}{w_{1}^{q}\left(y^{1 / q}(s)\right)} \\
& \forall n \in[0, K] \cap \mathbf{N} .
\end{aligned}
$$

Let $z_{2}(t)$ denote the function on the right-hand side of (41), which is a positive and nondecreasing function on $[0, K] \cap \mathbf{N}$. From (41), we have

$$
\begin{gathered}
z_{2}(0)=H_{1}(y(0))+\sum_{s=0}^{K-1} f(K) h^{q}(s) e^{-q t_{s}}, \\
y(n) \leq H_{1}^{-1}\left(z_{2}(n)\right) \\
\forall n \in[0, K] \cap \mathbf{N} .
\end{gathered}
$$

Using $\Delta z_{2}(n)=z_{2}(n+1)-z_{2}(n)$ and (42), we obtain

$$
\begin{gathered}
\Delta z_{2}(n)=f(K) e^{-q t_{n}} y(n)+g(K) e^{-q t_{n}} \frac{w_{2}^{q}\left(y^{1 / q}(n)\right)}{w_{1}^{q}\left(y^{1 / q}(n)\right)} \\
\leq f(K) e^{-q t_{n}} H_{1}^{-1}\left(z_{2}(n)\right) \\
+g(K) e^{-q t_{n}} \frac{w_{2}^{q}\left(\left(H_{1}^{-1}\left(z_{2}(n)\right)\right)^{1 / q}\right)}{w_{1}^{q}\left(\left(H_{1}^{-1}\left(z_{2}(n)\right)\right)^{1 / q}\right)}, \\
\forall n \in[0, K] \cap \mathbf{N} .
\end{gathered}
$$


From (43), we have

$$
\begin{aligned}
& \frac{\Delta z_{2}(n)}{H_{1}^{-1}\left(z_{2}(n)\right)} \\
& \quad \leq f(K) e^{-q t_{n}} \\
& \quad+g(K) e^{-q t_{n}} \frac{w_{2}^{q}\left(\left(H_{1}^{-1}\left(z_{2}(n)\right)\right)^{1 / q}\right)}{H_{1}^{-1}\left(z_{2}(n)\right) w_{1}^{q}\left(\left(H_{1}^{-1}\left(z_{2}(n)\right)\right)^{1 / q}\right)}
\end{aligned}
$$

for all $n \in[0, K] \cap \mathbf{N}$. Again by the mean-value theorem for integrals, for arbitrarily given integers $n, n+1 \in[0, K] \cap \mathbf{N}$, there exists $\xi$ in the open interval $\left(z_{2}(n), z_{2}(n+1)\right)$ such that

$$
\begin{aligned}
H_{2}\left(z_{2}(n+1)\right)-H_{2}\left(z_{2}(n)\right) & =\int_{z_{2}(n)}^{z_{2}(n+1)} \frac{d s}{H_{1}^{-1}(s)} \\
& =\frac{\Delta z_{2}(n)}{H_{1}^{-1}(\xi)} \leq \frac{\Delta z_{2}(n)}{H_{1}^{-1}\left(z_{2}(n)\right)}
\end{aligned}
$$

where $H_{2}$ is defined by (19). From (44) and (45), we have

$$
\begin{aligned}
& H_{2}\left(z_{2}(n+1)\right)-H_{2}\left(z_{2}(n)\right) \\
& \leq f(K) e^{-q t_{n}}+g(K) e^{-q t_{n}} \\
& \quad \times \frac{w_{2}^{q}\left(\left(H_{1}^{-1}\left(z_{2}(n)\right)\right)^{1 / q}\right)}{H_{1}^{-1}\left(z_{2}(n)\right) w_{1}^{q}\left(\left(H_{1}^{-1}\left(z_{2}(n)\right)\right)^{1 / q}\right)} .
\end{aligned}
$$

Taking $n=s$ in (46) and summing up over $s$ from 0 to $n-1$, from (46) we obtain

$$
\begin{aligned}
H_{2}\left(z_{2}(n)\right) & \\
\leq & H_{2}\left(z_{2}(0)\right)+\sum_{s=0}^{n-1} f(K) e^{-q t_{s}} \\
& +\sum_{s=0}^{n-1} g(K) e^{-q t_{s}} \frac{w_{2}^{q}\left(\left(H_{1}^{-1}\left(z_{2}(n)\right)\right)^{1 / q}\right)}{H_{1}^{-1}\left(z_{2}(s)\right) w_{1}^{q}\left(\left(H_{1}^{-1}\left(z_{2}(s)\right)\right)^{1 / q}\right)} \\
\leq & H_{2}\left(z_{2}(0)\right)+\sum_{s=0}^{K-1} f(K) e^{-q t_{s}} \\
& +\sum_{s=0}^{n-1} g(K) e^{-q t_{s}} \frac{w_{2}^{q}\left(\left(H_{1}^{-1}\left(z_{2}(n)\right)\right)^{1 / q}\right)}{H_{1}^{-1}\left(z_{2}(s)\right) w_{1}^{q}\left(\left(H_{1}^{-1}\left(z_{2}(s)\right)\right)^{1 / q}\right)}
\end{aligned}
$$

for all $n \in[0, K] \cap \mathbf{N}$. Let

$$
\begin{aligned}
z_{3}(n)= & H_{2}\left(z_{2}(0)\right)+\sum_{s=0}^{K-1} f(K) e^{-q t_{s}} \\
& +\sum_{s=0}^{n-1} g(K) e^{-q t_{s}} \frac{w_{2}^{q}\left(\left(H_{1}^{-1}\left(z_{2}(n)\right)\right)^{1 / q}\right)}{H_{1}^{-1}\left(z_{2}(s)\right) w_{1}^{q}\left(\left(H_{1}^{-1}\left(z_{2}(s)\right)\right)^{1 / q}\right)} .
\end{aligned}
$$

Then

$$
\begin{gathered}
z_{3}(0)=H_{2}\left(z_{2}(0)\right)+\sum_{s=0}^{K-1} f(K) e^{-q t_{s}}, \\
z_{2}(n) \leq H_{2}^{-1}\left(z_{3}(n)\right) .
\end{gathered}
$$

From (48) and (49), we have

$$
\begin{aligned}
& \frac{H_{1}^{-1}\left(H_{2}^{-1}\left(z_{3}(s)\right)\right) w_{1}^{q}\left(\left(H_{1}^{-1}\left(H_{2}^{-1}\left(z_{3}(s)\right)\right)\right)^{1 / q}\right) \Delta z_{3}(n)}{w_{2}^{q}\left(\left(H_{1}^{-1}\left(H_{2}^{-1}\left(z_{3}(n)\right)\right)\right)^{1 / q}\right)} \\
& \quad \leq g(K) e^{-q t_{n}} .
\end{aligned}
$$

Using the mean-value theorem for integrals, from (50) we have

$$
H_{3}\left(z_{3}(n)\right) \leq H_{3}\left(z_{3}(0)\right)+\sum_{s=0}^{n-1} g(K) e^{-q t_{s}}
$$

where $H_{3}$ is defined by (20). From (36), (42), (49), and (51), we have

$$
\begin{aligned}
z_{1}(n) \leq y(n) \leq H_{1}^{-1}\left(z_{2}(n)\right) \leq H_{1}^{-1}\left(H_{2}^{-1}\left(z_{3}(n)\right)\right) \\
\leq H_{1}^{-1}\left\{H_{2}^{-1}\left[H_{3}^{-1}\left(H_{3}\left(z_{3}(0)\right)+\sum_{s=0}^{n-1} g(K) e^{-q t_{s}}\right)\right]\right\} \\
=H_{1}^{-1}\left\{H _ { 2 } ^ { - 1 } \left[H _ { 3 } ^ { - 1 } \left(H_{3}\left(H_{2}\left(z_{2}(0)\right)+\sum_{s=0}^{K-1} f(K) e^{-q t_{s}}\right)\right.\right.\right. \\
\left.\left.\left.+\sum_{s=0}^{n-1} g(K) e^{-q t_{s}}\right)\right]\right\}
\end{aligned}
$$




$$
\begin{gathered}
=H_{1}^{-1} \times H_{2}^{-1}\left[H _ { 3 } ^ { - 1 } \left(H _ { 3 } \left(H _ { 2 } \left(H_{1}\left(z_{1}(0)\right)\right.\right.\right.\right. \\
\left.+\sum_{s=0}^{K-1} f(K) h^{q}(s) e^{-q t_{s}}\right) \\
\left.+\sum_{s=0}^{K-1} f(K) e^{-q t_{s}}\right) \\
\left.\left.\left.+\sum_{s=0}^{n-1} g(K) e^{-q t_{s}}\right)\right]\right\}, \\
\forall n \in[0, K] \cap \mathbf{N} .
\end{gathered}
$$

Using $v(n):=u^{q}(n)$ and (33), from (52) we obtain that

$u(n)$

$$
\begin{gathered}
=v^{1 / q}(n) \leq z_{1}^{1 / q}(n) \\
\leq\left\{H _ { 1 } ^ { - 1 } \left\{H _ { 2 } ^ { - 1 } \left[H _ { 3 } ^ { - 1 } \left(H _ { 3 } \left(H _ { 2 } \left(H_{1}\left(z_{1}(0)\right)\right.\right.\right.\right.\right.\right. \\
\left.+\sum_{s=0}^{K-1} f(K) h^{q}(s) e^{-q t_{s}}\right) \\
\left.+\sum_{s=0}^{K-1} f(K) e^{-q t_{s}}\right) \\
\left.\left.\left.\left.+\sum_{s=0}^{n-1} g(K) e^{-q t_{s}}\right)\right]\right\}\right\}^{1 / q}, \\
\forall n \in[0, K] \cap \mathbf{N} .
\end{gathered}
$$

Since $K$ is chosen arbitrarily, from (53) we have

$u(n)$

$$
\begin{gathered}
\leq\left\{H _ { 1 } ^ { - 1 } \left\{H _ { 2 } ^ { - 1 } \left[H _ { 3 } ^ { - 1 } \left(H _ { 3 } \left(H _ { 2 } \left(H_{1}\left(z_{1}(0)\right)\right.\right.\right.\right.\right.\right. \\
\left.+\sum_{s=0}^{n-1} f(n) h^{q}(s) e^{-q t_{s}}\right) \\
\left.+\sum_{s=0}^{n-1} f(n) e^{-q t_{s}}\right) \\
\left.\left.\left.\left.+\sum_{s=0}^{n-1} g(n) e^{-q t_{s}}\right)\right]\right\}\right\}^{1 / q}, \\
\forall n \in \mathbf{N}_{1} .
\end{gathered}
$$

This is our required estimation (16) of unknown function in (10).

\section{Application}

In this section, we apply our results to discuss the boundedness of solutions of an iterative difference equation with a weakly singular kernel.

Example 5. Suppose that $u(n)$ satisfies the difference equation

$$
\begin{aligned}
x(n)= & 1+\sum_{s=0}^{n-1}\left(t_{n}-t_{s}\right)^{-2 / 3} \tau_{s} \\
& \times\left[x(s)+\sum_{\tau=0}^{s-1}\left(t_{s}-t_{\tau}\right)^{-2 / 3} \tau_{\tau} x(\tau)\left(\ln |x(\tau)|^{4}\right)^{1 / 4}\right],
\end{aligned}
$$

$\forall n \in \mathbf{N}$,

where $t_{0}=0, \tau_{s}=t_{s+1}-t_{s}>0, \sup _{s \in \mathbf{N}, 0 \leq s \leq n-1}\left\{\tau_{s}, s \in \mathbf{N}\right\}=\tau$, and $\lim _{n \rightarrow \infty} t_{n}=\infty$. Then we have

$$
\begin{aligned}
|x(n)| \leq 1 & +\sum_{s=0}^{n-1}\left(t_{n}-t_{s}\right)^{-2 / 3} \tau_{s} \\
\times & {\left[|x(s)|+\sum_{\tau=0}^{s-1}\left(t_{s}-t_{\tau}\right)^{-2 / 3}\right.} \\
& \left.\times \tau_{\tau}|x(\tau)|\left(\ln |x(\tau)|^{4}\right)^{1 / 4}\right],
\end{aligned}
$$

for all $n \in \mathbf{N}$. Let $a(n) \equiv b(n) \equiv 1, h(n) \equiv 0, \beta=1 / 3, p=$ $4 / 3, q=4, w_{1}(u) \equiv 1, w_{2}(u)=u\left(\ln u^{4}\right)^{1 / 4}$. From (18) to $(20)$ we obtain that

$$
\begin{aligned}
& H_{1}(u):=\int_{0}^{u} d s=u, \quad u>0, \quad H_{1}(\infty)=\infty \\
& H_{1}^{-1}(u)=u \\
& H_{2}(u):=\int_{1}^{u} \frac{d s}{s}=\ln u, \quad u>0, \quad H_{2}(\infty)=\infty \\
& H_{2}^{-1}(u)=e^{u}, \\
& H_{3}(u):=\int_{1}^{u} \frac{d s}{s}=\ln u, \quad u>0, \quad H_{3}(\infty)=\infty \\
& H_{3}^{-1}(u)=e^{u}, \\
& f(n):=6^{3} \tau\left[\frac{e^{4 t_{n} / 3}}{(4 / 3)^{1 / 9}} \Gamma\left(\frac{1}{9}\right)\right]^{3}, \\
& g(n):=\tau\left(\frac{e^{4 t_{n} / 3}}{(4 / 3)^{1 / 9}} \Gamma\left(\frac{1}{9}\right)\right)^{3} .
\end{aligned}
$$


Using Theorem 4, we get

$$
\begin{aligned}
& u(n) \\
& \leq\left\{\operatorname { e x p } \left[\operatorname { e x p } \left(\operatorname { l n } \left(\ln 8+6^{3} \tau\left[\frac{e^{4 t_{n} / 3}}{(4 / 3)^{1 / 9}} \Gamma\left(\frac{1}{9}\right)\right]^{3}\right.\right.\right.\right. \\
& \left.\times \sum_{s=0}^{n-1} e^{-q t_{s}}\right) \\
& +\tau\left(\frac{e^{4 t_{n} / 3}}{(4 / 3)^{1 / 9}} \Gamma\left(\frac{1}{9}\right)\right)^{3} \\
& \left.\left.\left.\times \sum_{s=0}^{n-1} e^{-q t_{s}}\right)\right]\right\}^{1 / q}, \quad \forall n \in \mathbf{N},
\end{aligned}
$$

which is an upper bound of $|x(n)|$ in (55).

\section{Conflict of Interests}

The authors declare that there is no conflict of interests regarding the publication of this paper.

\section{Acknowledgments}

This research was supported by National Natural Science Foundation of China (Project no. 11161018), the NSF of Guangxi Zhuang Autonomous Region (no. 2012GXNSFAA053009), the high school specialty and curriculum integration project of Guangxi Zhuang Autonomous Region (no. GXTSZY2220), the Science Innovation Project of Department of Education of Guangdong province (Grant 2013KJCX0125), and the NSF of Guangdong Province (no. s2013010013385). The authors are very grateful to the editor and the referees for their careful comments and valuable suggestions on this paper.

\section{References}

[1] I. A. Bihari, "A generalization of a lemma of Bellman and its application to uniqueness problems of differential equations," Acta Mathematica Academiae Scientiarum Hungaricae, vol. 7, pp. 81-94, 1956.

[2] D. Henry, Geometric Theory of Semilinear Parabolic Equations, vol. 840, Springer, Berlin, Germany, 1981.

[3] D. Bainov and P. Simeonov, Integral Inequalities and Applications, vol. 57, Kluwer Academic, Dordrecht, The Netherlands, 1992.

[4] M. Medved', "A new approach to an analysis of Henry type integral inequalities and their Bihari type versions," Journal of Mathematical Analysis and Applications, vol. 214, no. 2, pp. 349366, 1997.

[5] B. G. Pachpatte, Inequalities for Differential and Integral Equations, vol. 197, Academic Press, London, UK, 1998.

[6] I. Podlubny, Fractional Differential Equations, vol. 198, Academic Press, New York, NY, USA, 1999.

[7] M. Medved', "Nonlinear singular integral inequalities for functions in two and $n$ independent variables," Journal of Inequalities and Applications, vol. 5, no. 3, pp. 287-308, 2000.
[8] E. H. Yang, "A new nonlinear discrete inequality and its application," Annals of Differential Equations. Weifen Fangcheng Niankan, vol. 17, no. 3, pp. 261-267, 2001.

[9] O. Lipovan, "A retarded Gronwall-like inequality and its applications," Journal of Mathematical Analysis and Applications, vol. 252, no. 1, pp. 389-401, 2000.

[10] W. Zhang and S. Deng, "Projected Gronwall-Bellman's inequality for integrable functions," Mathematical and Computer Modelling, vol. 34, no. 3-4, pp. 393-402, 2001.

[11] Q. H. Ma and E. H. Yang, "Estimates on solutions of some weakly singular Volterra integral inequalities," Acta Mathematicae Applicatae Sinica. Yingyong Shuxue Xuebao, vol. 25, no. 3, pp. 505-515, 2002 (Chinese).

[12] F. W. Meng and W. N. Li, "On some new nonlinear discrete inequalities and their applications," Journal of Computational and Applied Mathematics, vol. 158, no. 2, pp. 407-417, 2003.

[13] R. P. Agarwal, S. Deng, and W. Zhang, "Generalization of a retarded Gronwall-like inequality and its applications," Applied Mathematics and Computation, vol. 165, no. 3, pp. 599-612, 2005.

[14] B.-I. Kim, "On some Gronwall type inequalities for a system integral equation," Bulletin of the Korean Mathematical Society, vol. 42, no. 4, pp. 789-805, 2005.

[15] B. G. Pachpatte, "On certain nonlinear integral inequalities involving iterated integrals," Tamkang Journal of Mathematics, vol. 37, no. 3, pp. 261-271, 2006.

[16] W. S. Cheung, "Some new nonlinear inequalities and applications to boundary value problems," Nonlinear Analysis. Theory, Methods \& Applications A: Theory and Methods, vol. 64, no. 9, pp. 2112-2128, 2006.

[17] W. S. Wang, "A generalized retarded Gronwall-like inequality in two variables and applications to BVP," Applied Mathematics and Computation, vol. 191, no. 1, pp. 144-154, 2007.

[18] Q. H. Ma and W. S. Cheung, "Some new nonlinear difference inequalities and their applications," Journal of Computational and Applied Mathematics, vol. 202, no. 2, pp. 339-351, 2007.

[19] M. Medved', "On singular versions of Bihari and WendroffPachpatte type integral inequalities and their application," Tatra Mountains Mathematical Publications, vol. 38, pp. 163-174, 2007.

[20] H. Ye, J. Gao, and Y. Ding, "A generalized Gronwall inequality and its application to a fractional differential equation," Journal of Mathematical Analysis and Applications, vol. 328, no. 2, pp. 1075-1081, 2007.

[21] A. Abdeldaim and M. Yakout, "On some new integral inequalities of Gronwall-Bellman-Pachpatte type," Applied Mathematics and Computation, vol. 217, no. 20, pp. 7887-7899, 2011.

[22] T. H. Gronwall, "Note on the derivatives with respect to a parameter of the solutions of a system of differential equations," Annals of Mathematics, vol. 20, no. 4, pp. 292-296, 1919.

[23] R. Bellman, "The stability of solutions of linear differential equations," Duke Mathematical Journal, vol. 10, pp. 643-647, 1943.

[24] T. E. Hull and W. A. J. Luxemburg, "Numerical methods and existence theorems for ordinary differential equations," Numerische Mathematik, vol. 2, pp. 30-41, 1960.

[25] D. Willett and J. S. W. Wong, "On the discrete analogues of some generalizations of Gronwall's inequality," vol. 69, pp. 362-367, 1965.

[26] S. Sugiyama, "On the stability problems of difference equations," Bulletin of Science and Engineering Research Laboratory. Waseda University, vol. 45, pp. 140-144, 1969. 
[27] B. G. Pachpatte, "Finite difference inequalities and discrete time control systems," Indian Journal of Pure and Applied Mathematics, vol. 9, no. 12, pp. 1282-1290, 1978.

[28] M. Kuczma, An Introduction to the Theory of Functional Equations and Inequalities: Cauchys Equation and Jensens Inequality, vol. 489, University of Katowice, Katowice, Poland, 1985.

[29] W.-S. Cheung and J. Ren, "Discrete non-linear inequalities and applications to boundary value problems," Journal of Mathematical Analysis and Applications, vol. 319, no. 2, pp. 708-724, 2006.

[30] E. H. Yang, Q. H. Ma, and M. C. Tan, "Discrete analogues of a new class of nonlinear Volterra singular integral inequalities," Journal of Jinan University, vol. 28, no. 1, pp. 1-6, 2007.

[31] K. Zheng, S. Zhong, and M. Ye, "Discrete nonlinear inequalities in time control systems," in Proceedings of the International Conference on Apperceiving Computing and Intelligence Analysis (ICACIA '09), pp. 403-406, October 2009.

[32] K. Zheng, H. Wang, and C. Guo, "On nonlinear discrete weakly singular inequalities and applications to Volterra-type difference equations," Advances in Difference Equations, vol. 2013, p. 239, 2013. 


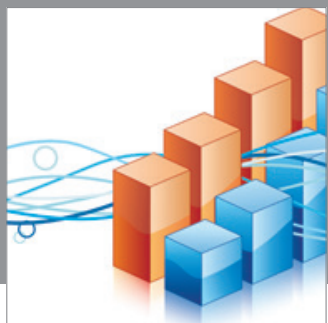

Advances in

Operations Research

mansans

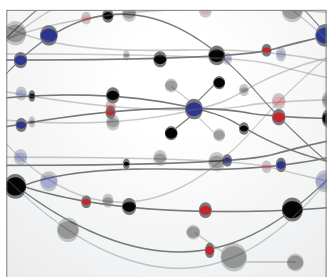

The Scientific World Journal
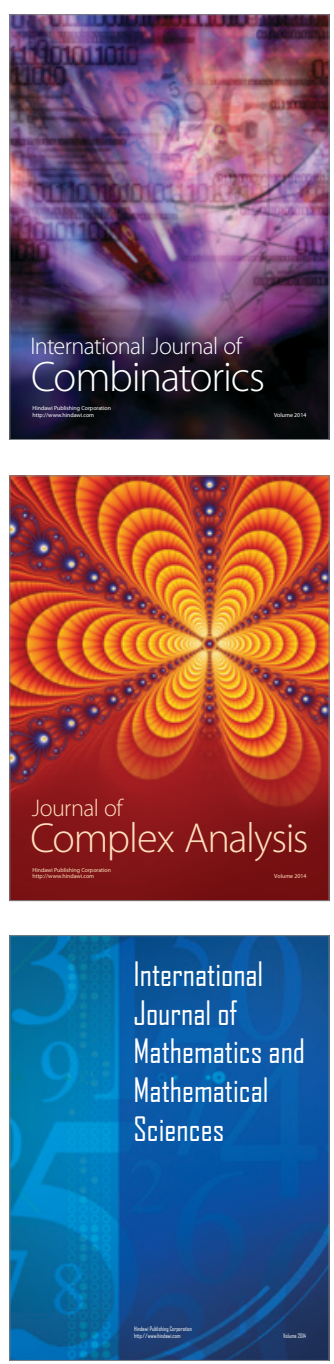
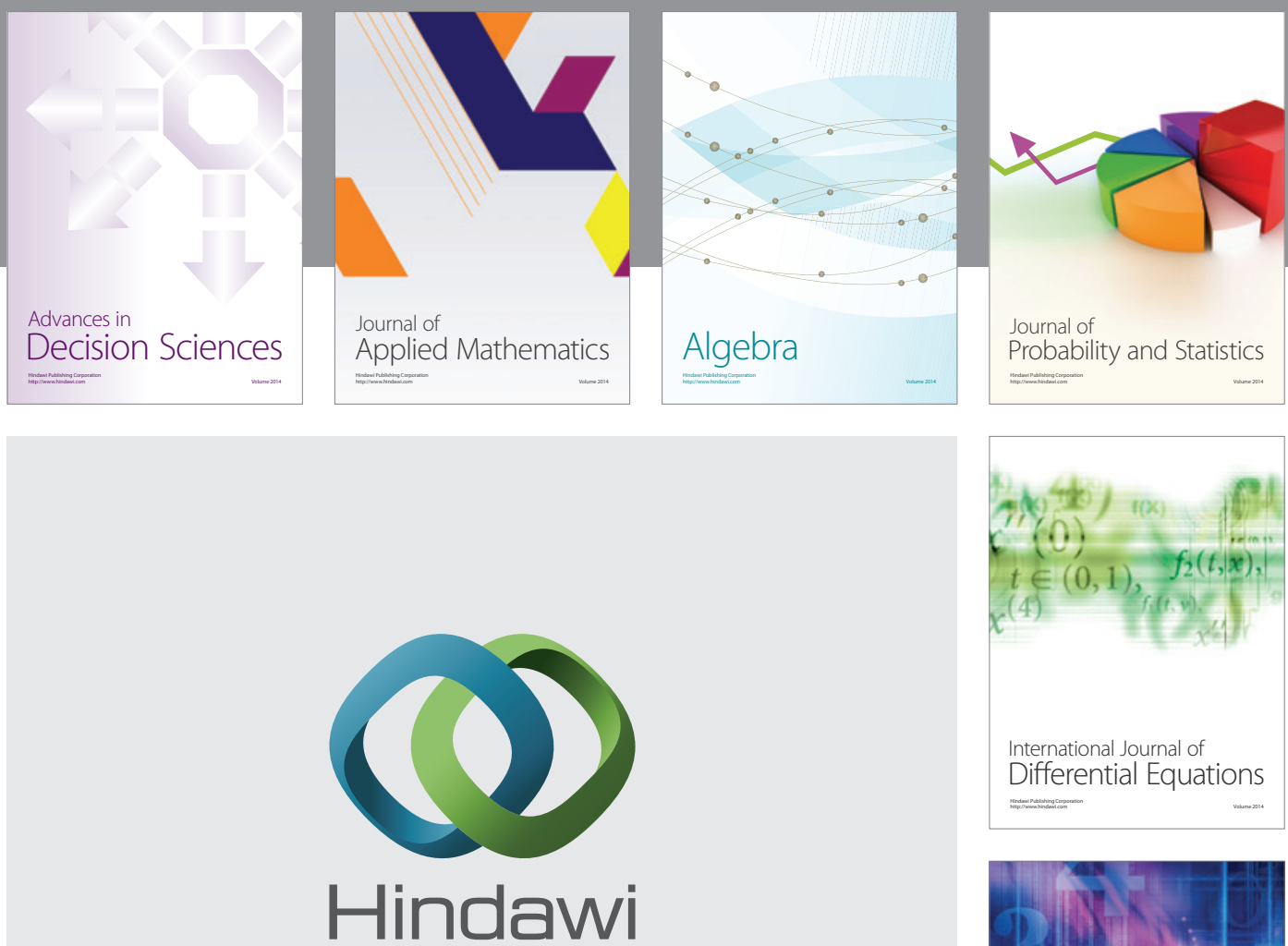

Submit your manuscripts at http://www.hindawi.com
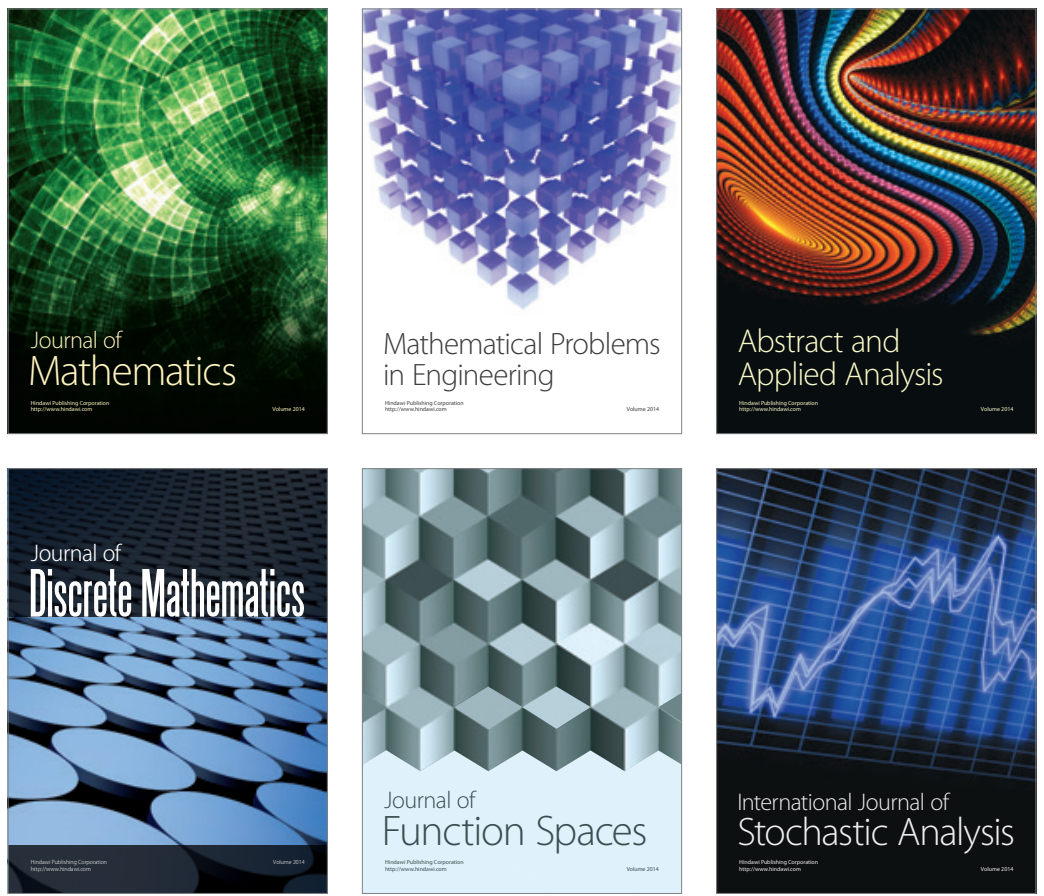

Journal of

Function Spaces

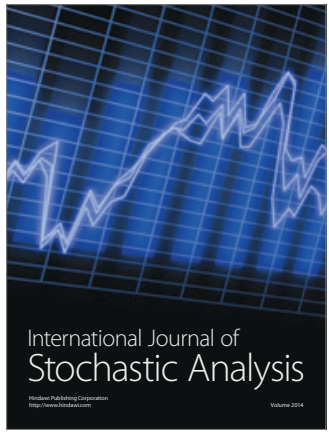

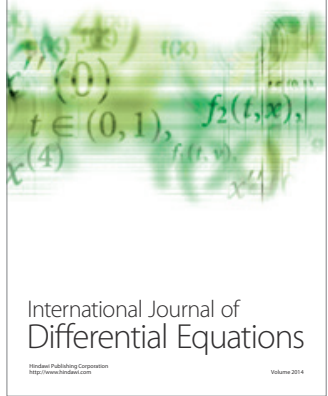
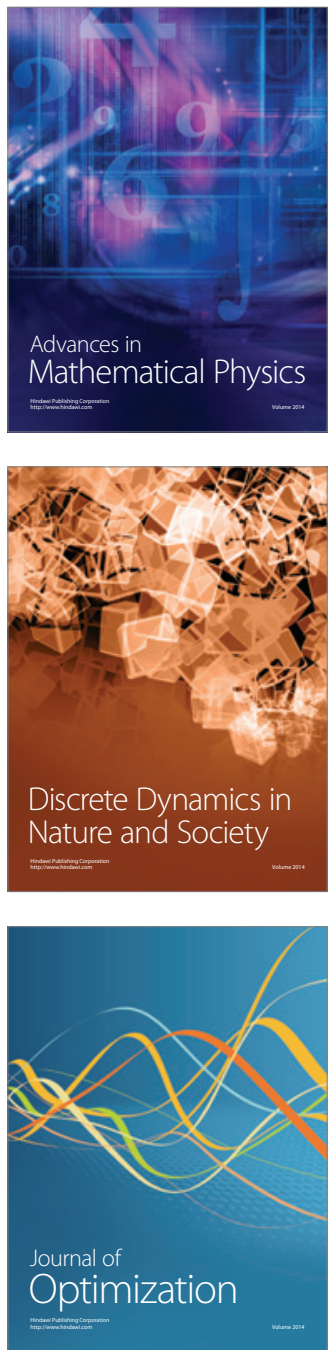